\title{
Puncture of a Disc and Application of Nucleus Pulposus Induces Disc Herniation-Like Changes and Osteophytes. An Experimental Study in Rats
}

\author{
Kjell Olmarker ${ }^{*}$
}

\author{
Muskuloskeletal Research, Department of Medical chemistry and Cellbiology, Institute of Biomedicine, Sahlgrenska \\ Academy, University of Gothenburg, Gothenburg, Sweden
}

\begin{abstract}
It has been observed that puncture of a lumbar disc may induce formation of a nodule on the surface of the disc and osteophytes. It is not known if this is based on the presence of a foreign tissue or specifically by the presence of nucleus pulposus or on the disc injury. In this study these mechanisms were separated by comparing disc puncture with application of nucleus pulposus without disc injury, with superficial disc injury without nucleus pulposus and with application of fat. Fifty rats underwent facetectomy of the left L4-5 facet. Ten additional rats were used as donor rats. The rats were exposed to disc puncture $(n=10)$, application of homologous nucleus pulposus $(n=10)$, application of homologous fat tissue $(n=10)$, superficial disc injury $(n=10)$ and ten rats served as control. After 3 weeks the rats were examined macroscopically regarding presence of disc nodules and osteophytes. A limited histological analysis was performed to obtain a microscopic overview of any observed changes. In rats with application of fat, superficial disc injury and in sham controls there were almost no changes observed. However, in rats with disc puncture and applied nucleus pulposus there were clear disc nodules and osteophytes noted. Microscopically the nodules comprised granulation tissue and the osteophytes cortical bone. In conclusion, the data indicate that the presence of nucleus pulposus is more likely to be responsible for the formation of disc nodules and osteophytes than disc injury or the presence of a foreign tissue. This may provide new insights in the mechanisms regarding the formation of disc herniations and osteophytes.
\end{abstract}

Keywords: Spine, disc herniation, nucleus pulposus, disc injury, osteophytes, rat.

\section{INTRODUCTION}

It is well established that herniations of the intervertebral discs are closely related to the radiating pain in the lower limb called sciatica [1]. However, the temporal relationship between onset of symptoms and the event of herniation is not known. It seems evident that the disc tissue must be more or less viscous at the time of herniation and one may suspect that this tissue may not be able to compress a nerve root. The hard tissue forming the disc herniation nodule that is seen at discectomy, and that may be able to compress a nerve root, does not have the physical properties to herniate from the centre of the disc. It has previously been presented that disc puncture in the rat may induce a leakage of nucleus pulposus that is liquefied within the first minute of "herniation" and did not produce any visible nodule [2]. However, some weeks after the disc puncture, a clear visible "nodule" was present at the location of the puncture. One may therefore assume that the viscous or liquid herniating nucleus pulposus material is later subject to fibrotic changes that will render it harder. In such case, a manifest disc herniation may thus be of earlier date than the onset of symptoms. However, one may also consider the possibility that disc-derived bioactive substances from the nucleus pulposus or from the disc injury may initiate reactive changes at the surface of the disc and

*Address correspondence to this author at the Muskuloskeletal Research, Department of Medical chemistry and Cellbiology, Institute of Biomedicine, Sahlgrenska Academy, University of Gothenburg, SE-405 30 Gothenburg, Sweden; Tel: +46-31-7866660; E-mail.: kjell.olmarker@gu.se adjacent bone. In this study these mechanisms were separated by comparing disc puncture with application of nucleus pulposus without disc injury, with superficial disc injury without nucleus pulposus herniation and with application of fat.

\section{MATERIAL AND METHODS}

A total of 60 Sprague-Dawley rats weighing 200-250g were anaesthetized with isoflurane inhalation (Baxter Medical AB, Kista, Sweden). In 50 rats, the left facet joint between the 4th and the 5th lumbar vertebra was removed and the 4th lumbar dorsal root ganglion and the 5th lumbar nerve root, including the intervertebral disc between the 4th and 5 th lumbar vertebrae, were visualized. Ten rats served as tissue donors.

Sham, $(n=10)$ : No further procedures were performed in this group.

Application of homologous fat tissue, $(n=10)$ : Approximately $1 \mathrm{mg}$ of peritoneal fat harvested from the intra abdominal omentum from a donor rat was applied over the dorsolateral aspect of the L 4-5 disc.

Superficial disc injury, $(\mathrm{n}=10)$ : The outer layer of the annulus fibrosus of the L 4-5 disc was gently scratched with an $0.4 \mathrm{~mm}$ diameter injection needle. Great care was taken not to penetrate the annulus fibrosus in order to avoid leakage of nucleus pulposus. 
Application of homologous nucleus pulposus, $(\mathrm{n}=10)$ : Nucleus pulposus from one tail disc from a donor rat (appr. 1 $\mathrm{mg}$ ) was applied onto the L4-5 disc.

Disc puncture, $(\mathrm{n}=10)$ : The L4-5 disc was punctured using a $0.4 \mathrm{~mm}$ diameter injection needle. After injecting some air into the disc space the puncture typically induced a leakage of nucleus pulposus out into the spinal canal.

After the specific procedure the spinal muscles were sutured and the skin closed by metal-clips.

The rats were killed after 3 weeks. Changes in the spinal canal at the puncture site were evaluated macroscopically via the operation microscope and by probing regarding; inflammation, healing of the disc injury when applicable, height and consistency of any nodule over the site of disc puncture, and height of osteophytes on the vertebrae adjacent to the disc. The findings were graded according to a semiquantitative scale. The evaluations were performed in a blinded fashion.

In selected cases, the spinal segment, including the two adjacent vertebrae, was removed en bloc to obtain a microscopic overview of the observed changes. The specimens were decalcified by 15\% EDTA (Sigma-Aldrich, St. Louis, USA). Cross-sections of the disc and the part of the L4 vertebra displaying osteophytes were stained by HtxEosin and assessed using light microscopy.

The study was approved by the local animal ethics committee.

\section{RESULTS}

The rats seemed to tolerate the surgery well and did not display any pain as assessed by the naked eye. The data from the macroscopic evaluation are presented in Table 1.

Sham: Two animals displayed slight changes on the disc surface. One of these animals showed a minor osteophyte and also pronounced inflammation (Fig. 1).

Application of homologous fat tissue: One animal showed slight changes on the disc surface and a minor osteophyte. No inflammatory reactions were noted.

Superficial disc injury: There was no inflammation, nodules or osteophytes noted. The disc injury was completely healed in all animals.

Application of homologous nucleus pulposus: There were slight to pronounced inflammation in the animals. Disc nodules were found in all animals, and osteophytes were found in all but two animals.

Disc puncture: Changes in inflammation, disc nodule consistency and osteophytes were marginally more pronounced compared to the animals with applied nucleus pulposus. Disc nodule height was similar to the nucleus pulposus application group. However, one animal showed no changes in any of the parameters.

The light microscopic evaluation showed that the nodules found in the punctured animals and animals with applied nucleus pulposus comprised collagen and various cell-types (Fig. 2). These were mainly fibroblasts but also inflammatory cells and chondrocyte-like cells were

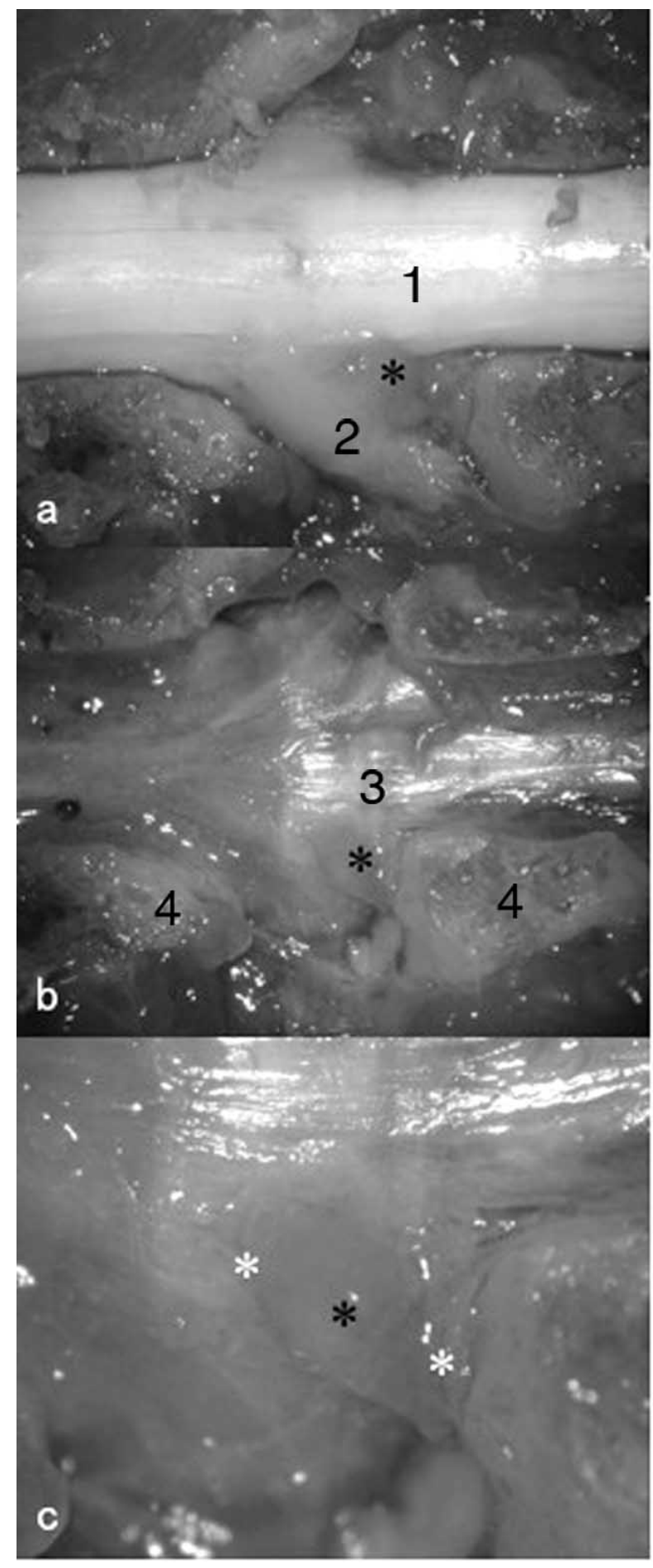

Fig. (1). Spinal canal 3 weeks after disc puncture. a) The gap between the thecal sac (1) and the $4^{\text {th }}$ lumbar dorsal root ganglion and spinal nerve (2) has been filled with an elastic nodule (asterisk). b) The nodule as seen when the nerve tissue has been removed (asterisk). The nodule is located at the same level as the intervertebral disc (3). The cut pedicles of the $4^{\text {th }}$ (left) and $5^{\text {th }}$ lumbar vertebrae (4) are indicated for orientation. c) Close-up of the nodule (black asterisk). Osteophyte formation may be noted on the $4^{\text {th }}$ (left) and the $5^{\text {th }}$ lumbar vertebrae adjacent to the disc (white asterisks). (The diameter of the thecal sac is approximately $2,5 \mathrm{~mm}$ for size orientation). 
Table 1. Macroscopic Findings

\begin{tabular}{|c|c|c|c|c|}
\hline \multicolumn{5}{|l|}{ Sham } \\
\hline I & $\mathrm{H}$ & Disc h. & Disc c. & Osteophyte \\
\hline++ & N/A & $(+)$ & $(+)$ & $(+)$ \\
\hline 0 & N/A & 0 & 0 & 0 \\
\hline 0 & N/A & 0 & 0 & 0 \\
\hline 0 & $\mathrm{~N} / \mathrm{A}$ & 0 & 0 & 0 \\
\hline 0 & N/A & 0 & 0 & 0 \\
\hline 0 & $\mathrm{~N} / \mathrm{A}$ & 0 & 0 & 0 \\
\hline 0 & N/A & $(+)$ & $(+)$ & 0 \\
\hline 0 & N/A & 0 & 0 & 0 \\
\hline 0 & N/A & 0 & 0 & 0 \\
\hline 0 & $\mathrm{~N} / \mathrm{A}$ & 0 & 0 & 0 \\
\hline \multicolumn{5}{|c|}{ Application of Fat } \\
\hline 0 & N/A & 0 & 0 & 0 \\
\hline 0 & N/A & 0 & 0 & 0 \\
\hline 0 & N/A & 0 & 0 & 0 \\
\hline 0 & $\mathrm{~N} / \mathrm{A}$ & $(+)$ & + & $(+)$ \\
\hline 0 & N/A & 0 & 0 & 0 \\
\hline 0 & N/A & 0 & 0 & 0 \\
\hline 0 & N/A & 0 & 0 & 0 \\
\hline 0 & N/A & 0 & 0 & 0 \\
\hline 0 & $\mathrm{~N} / \mathrm{A}$ & 0 & 0 & 0 \\
\hline 0 & N/A & 0 & 0 & 0 \\
\hline \multicolumn{5}{|c|}{ Superficial Disc Injury } \\
\hline 0 & + & 0 & 0 & 0 \\
\hline 0 & + & 0 & 0 & 0 \\
\hline 0 & + & 0 & 0 & 0 \\
\hline 0 & + & 0 & 0 & 0 \\
\hline 0 & + & 0 & 0 & 0 \\
\hline 0 & + & 0 & 0 & 0 \\
\hline 0 & + & 0 & 0 & 0 \\
\hline 0 & + & 0 & 0 & 0 \\
\hline 0 & + & 0 & 0 & 0 \\
\hline 0 & + & 0 & 0 & 0 \\
\hline \multicolumn{5}{|c|}{ Application of Nucleus Pulposus } \\
\hline+ & $\mathrm{N} / \mathrm{A}$ & + & + & + \\
\hline+ & $\mathrm{N} / \mathrm{A}$ & ++ & + & $(+)$ \\
\hline$(+)$ & $\mathrm{N} / \mathrm{A}$ & ++ & $(+)$ & 0 \\
\hline$(+)$ & $\mathrm{N} / \mathrm{A}$ & ++ & $(+)$ & $(+)$ \\
\hline++ & N/A & ++ & $(+)$ & + \\
\hline$(+)$ & N/A & ++ & $(+)$ & 0 \\
\hline$(+)$ & $\mathrm{N} / \mathrm{A}$ & + & $(+)$ & $(+)$ \\
\hline$(+)$ & $\mathrm{N} / \mathrm{A}$ & $(+)$ & + & $(+)$ \\
\hline$(+)$ & N/A & ++ & + & + \\
\hline$(+)$ & N/A & + & + & + \\
\hline
\end{tabular}




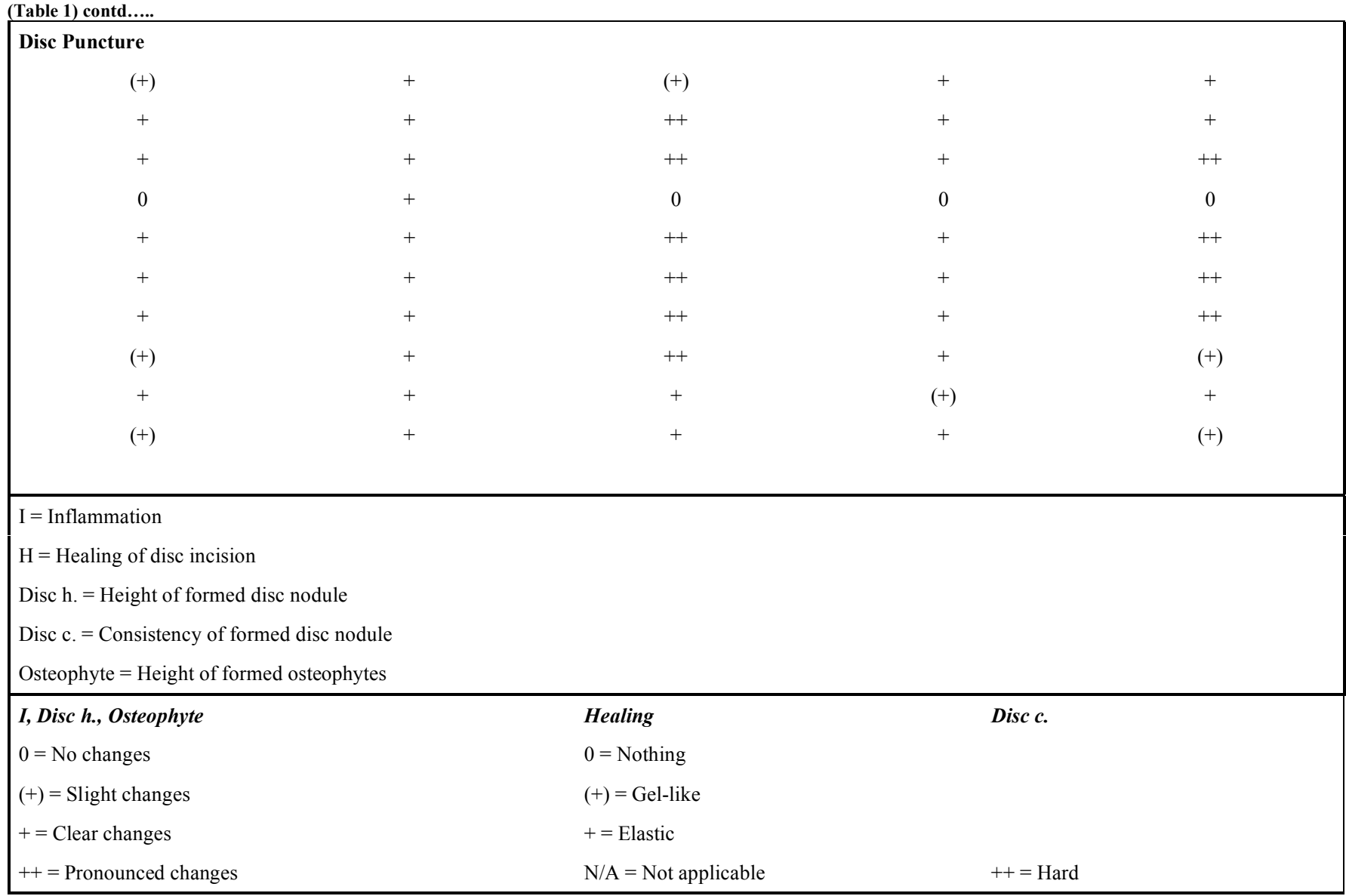

identified. The tissue was defined as granulation tissue before remodelling. These were consistent findings in all analysed specimens. The osteophytes comprised thickening of the cortical bone (Fig. 3).

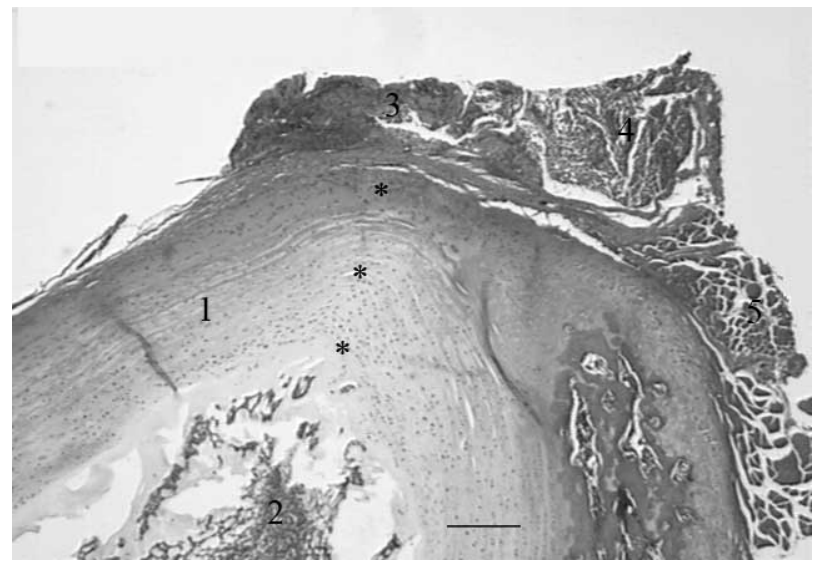

Fig. (2). Cross-section of a rat disc 3 weeks after disc puncture. The dorsolateral aspect of the disc is upwards in the picture. The dorsal surface of the disc is upper left and the ventral aspect is lower right. The annulus fibrosus (1) is surrounding the nucleus pulposus (2). A nodule (3) has been formed over the incision channel, which is indicated by asterisks. The nodule is just medial to the nerve root

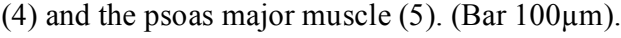

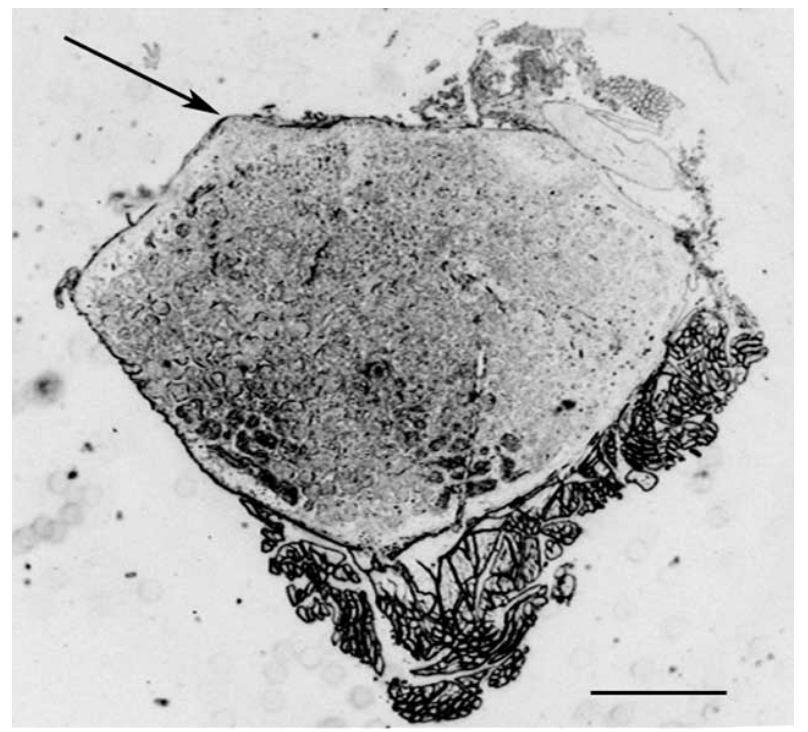

Fig. (3). Cross-section of a rat lumbar vertebra displaying a newly formed osteophyte (arrow) on the dorsolateral aspect. (Bar $1 \mathrm{~mm}$ ).

\section{DISCUSSION}

Disc puncture and application of nucleus pulposus induced a nodule on the disc surface and osteophytes, whereas application of fat, an incomplete injury to the annulus fibrosus or the facetectomy per se did not. A limited histological analysis noted that the nodules comprised 
granulation tissue with collagen and various cell types and that the osteophytes comprised cortical bone.

To be able to herniate through a ruptured annulus fibrosus the nucleus pulposus has to be more or less viscous. This is in contrast to the elastic or hard tissue that is encountered at surgery and termed a "disc herniation" and that may compress or displace intra spinal nervous tissue. Nevertheless, it is likely that this latter tissue is related to a leakage from the intervertebral disc. It is, however, difficult to understand how this hard tissue has been able to penetrate through a presumably narrow injury in the intervertebral disc.

It has previously been observed that disc puncture in the rat may result in disc herniation-like nodules on the disc surface and osteophytes on adjacent vertebra [2]. One may assume that the tissue is the result either of fibrotic organisation of the herniated disc material or of the activity of nucleus pulposus-related bioactive substances. As previously described, the leaking nucleus pulposus is liquefied within the first minute after disc puncture [2]. It is therefore less likely that the observed nodules is the result of fibrotic changes of the herniated material since the nucleus pulposus has "melted" away and there is no tissue left in which fibrotic changes might occur. This is supported by the observation that fat application did not result in any noduleformation. It seems more likely that any foreign material placed on the disc surface is resorbed.

Contrary, nodules and osteophytes appeared after disc puncture and application of nucleus pulposus. This may suggest that the changes are more likely to be the result of the presence of bioactive substances derived from the nucleus pulposus. It is not likely, however, that the disc injury per se may initiate such activity since an incomplete injury of the annulus fibrosus with no nucleus pulposus leakage did not induce nodule and osteophyte formation.

It has previously been described that the presence of osteophytes is related to experimental disc injury and clinical disc degeneration [3-6]. It is also known that degenerated discs often have "annular tears" which theoretically may allow leakage of substances from the nucleus pulposus out into the spinal canal [7-9]. Based on the findings in the present study, one may therefore suspect that one mechanism for osteophyte formation in relation to disc degeneration may be due to leakage of bioactive substances from tears in the degenerated disc.

In a previous study it was observed that application of nucleus pulposus onto a dorsal root ganglion in the rat resulted in a characteristic semi-lunar swelling of the ganglion capsule [10]. This swelling was probably induced by TNF from the nucleus pulposus since a later experiment demonstrated that treatment with a selective TNF alphainhibitor reduced the formation of this swelling [11]. One may possibly assume that there may be similar mechanisms present in this study on the dorsal root ganglion as in the present study.

It may be noted that the histologic evaluation was performed only in order to obtain a preliminary microscopic overview of the observed changes. A more detailed assessment including immunohistologic techniques and chemical characterisation must be performed in order to define the structural components of the observed changes.

The data from the present study thus seem to indicate that osteophytes and the nodule on the disc surface, that might possibly be analogous to a disc herniation, may be induced as the result of bioactive substances released from the nucleus pulposus. It may be argued, however, that the physical properties between the nucleus pulposus in young rats is different from human adult degenerated nucleus pulposus in the sense that the rat nucleus pulposus is gel-like and the human nucleus pulposus is degenerated and fibrotic to certain degrees. One could argue that a fibrotic tissue would indeed form a hard tissue following herniation. However, it must be considered that a fibrotic tissue will be less likely to herniate. Instead, one might suspect that a fibrotic nucleus pulposus would rather remain inside the annulus fibrosus but that there may be a leakage of fluid comprising the suggested bioactive substances that in turn may initiate changes on the disc surface that eventually will result in the nodules found in the present study. These nodules may thus be the origin of the "disc herniations" found at surgery and observed radiologically.

In conclusion, the data in the present study show that nodules on the disc surface and osteophytes may be formed in the presence of nucleus pulposus but not following application of fat or by superficial annulus fibrosus injury. This preliminary study may provide new insights in the mechanisms regarding the formation of a disc herniation and osteophytes.

\section{ACKNOWLEDGEMENTS}

This work was supported by grants from the Swedish Research Council (521-2007-2956), the Gothenburg Medical Society, AFA Insurance, the Gothenburg Medical Society, the Ollie and Elof Ericsson Foundation for Scientific Research, Stiftelsen Olle Engkvist byggmästare, the IngaBritt and Arne Lundbergs Foundation and the Felix Neubergh Foundation.

\section{ABBREVIATION}

EDTA $=$ Ethylenediaminetetraacetic acid

\section{REFERENCES}

[1] Mixter WJ, Barr JS. Rupture of the intervertebral disc with involvement of the spinal canal. N Engl J Med 1934; 211: 210-5.

[2] Olmarker K. Inflammatory mechanisms in disc related pain. In: Lindgren $\mathrm{K}$, editor. LBP, controversies in clinical practice and research. Helsinki: ORTON 2007; p. 81-7.

[3] Lipson SJ, Muir H. Vertebral osteophyte formation in experimental disc degeneration. Morphologic and proteoglycan changes over time. Arthritis Rheum 1980; 23(3): 319-24.

[4] Vernon-Roberts B, Pirie CJ. Degenerative changes in the intervertebral discs of the lumbar spine and their sequelae. Rheumatol Rehabil 1977; 16(1): 13-21.

[5] Pye SR, Reid DM, Adams JE, Silman AJ, O'Neill TW. Radiographic features of lumbar disc degeneration and bone mineral density in men and women. Ann Rheum Dis 2006; 65(2): 234-8.

[6] Pye SR, Reid DM, Lunt M, Adams JE, Silman AJ, O'Neill TW. Lumbar disc degeneration: association between osteophytes, endplate sclerosis and disc space narrowing. Ann Rheum Dis 2007; 66(3): 330-3.

[7] Rydevik B, Brown MD, Lundborg G. Pathoanatomy and pathophysiology of nerve root compression. Spine 1984; 9(1): 715 . 
[8] Loneragan R, Khangure MS, McCormick C, Hardcastle P. Comparison of magnetic resonance imaging and computed tomographic discography in the assessment of lumbar disc degeneration. Aust Radiol 1994; 38(1): 6-9.

[9] Hadjipavlou AG, Simmons JW, Pope MH, Necessary JT, Goel VK. Pathomechanics and clinical relevance of disc degeneration and annular tear: a point-of-view review. Am J Orthop 1999; 28(10): $561-71$.
[10] Murata Y, Rydevik B, Takahashi K, Larsson K, Olmarker K. Incision of the intervertebral disc induces disintegration and increases permeability of the dorsal root ganglion capsule. Spine 2005; 30(15): 1712-6.

[11] Murata Y, Onda A, Rydevik B, Takahashi I, Takahashi K, Olmarker K. Changes in pain behavior and histologic changes caused by application of tumor necrosis factor-alpha to the dorsal root ganglion in rats. Spine 2006; 31(5): 530-5.

(C) Kjell Olmarker; Licensee Bentham Open.

This is an open access article licensed under the terms of the Creative Commons Attribution Non-Commercial License (http://creativecommons.org/licenses/by-nc/3.0/) which permits unrestricted, non-commercial use, distribution and reproduction in any medium, provided the work is properly cited. 